\title{
Dynamics of excimer laser-ablated aluminum neutral atom plume measured by dye laser resonance absorption photography
}

\author{
Ronald M. Gilgenbach and Peter L. G. Ventzek \\ Intense Energy Beam Interaction Laboratory, Nuclear Engineering Department, University of Michigan, \\ Ann Arbor, Michigan 48109-2104
}

(Received 9 November 1990; accepted for publication 13 February 1991)

\begin{abstract}
We report the first dye laser resonance absorption photographs of a single species of aluminum ground-state neutral atoms in the plume ablated from solid aluminum by $\mathrm{KrF}$ excimer laser radiation. Aluminum ground-state neutral atoms were diagnosed by illuminating the ablated plume with a dye laser tuned to the $3^{2} P_{1 / 2}-4^{2} S_{1 / 2}$ transition at 394.4 $\mathrm{nm}$. Measurements have been performed in vacuum as well as in argon and air environments. Streaming velocities measured for neutral aluminum atoms in vacuum ranged from $0.5 \times 10^{6} \mathrm{~cm} / \mathrm{s}$ at low excimer laser fluences of $1-2 \mathrm{~J} / \mathrm{cm}^{2}$ to $3.4 \times 10^{6} \mathrm{~cm} / \mathrm{s}$ at high fluences of $7 \mathrm{~J} / \mathrm{cm}^{2}$. Dye laser resonance absorption photography measurements of ablated aluminum in argon and air showed slower expansion at 50 and 200 Torr, while observations at 760 Torr indicate turbulent mixing of aluminum neutrals near the surface. Differences between data in argon and air may be due to oxidation of neutral aluminum atoms.
\end{abstract}

Excimer laser ablation has significant potential for both etching of polymeric photoresist and deposition of thin films in microelectronics manufacturing. Measurement of neutral atom expansion in vacuum is important to these and other applications, including flashover in high voltage equipment. A number of diagnostics have been investigated for time-resolved measurements of laser-ablated density, ${ }^{1-3}$ composition, ${ }^{4,5}$ hydrodynamics, ${ }^{5-9}$ temperature, ${ }^{4.5}$ and streaming velocity. ${ }^{1-12}$ In previous experiments elsewhere, laser-induced fluorescence measurements ${ }^{4}$ gave species-resolved velocity distributions of $\mathrm{Al}$ and $\mathrm{AlO}$ from excimer laser-ablated alumina $\left(\mathrm{Al}_{2} \mathrm{O}_{3}\right)$ in vacuum. Others have utilized spectroscopic time-of-flight (TOF) to measure velocities of ablated emissive ions and neutrals of $\mathrm{Y}$, $\mathrm{Ba}, \mathrm{Cu}$, and $\mathrm{O} .{ }^{9-12} \mathrm{~A}$ recent experiment reported TOF measurements of velocities for laser-ablated nonemissive ground-state neutrals and ions of $\mathrm{Y}, \mathrm{Ba}$, and $\mathrm{Cu}$ using transient absorption spectroscopy by means of a Xe lamp, spectrometer, and photomultiplier. ${ }^{5}$

In this letter, we report the first direct photographic measurements of excimer laser-ablated ground-state neutral aluminum plume dynamics by dye laser resonance $a b-$ sorption (DLRA) photography. Experiments reported here were performed in vacuum, argon, and air. The DLRA photography technique has a number of advantages as a laser ablation diagnostic: (1) It detects only a single species of nonemissive particle; (2) the sensitivity is very high; (3) the dynamics of the selected species over the entire plume is measured in a single ( $20 \mathrm{~ns}$ ) laser pulse; and (4) it is applicable in vacuum as well as gaseous and plasma environments.

The experimental configuration is depicted in Fig. 1. A $\mathrm{KrF}$ excimer laser generates a maximum of $1.5 \mathrm{~J}$ per pulse at $248 \mathrm{~nm}$ wavelength. The excimer laser beam is attenuated by quartz plates and focused by a $30 \mathrm{~cm}$ focal length lens to a $0.04 \mathrm{~cm}^{2}$ spot size on an aluminum target at normal incidence. An expanded dye laser beam passes through the ablation plume parallel to the surface at $90^{\circ}$ to the excimer laser beam. The $\mathrm{XeCl}$ laser-pumped dye laser has a pulse length of $20 \mathrm{~ns}$ and a linewidth of $0.2 \mathrm{~cm}^{-1}$. The relative delay between the $40 \mathrm{~ns} \mathrm{KrF}$ laser pulse and the dye laser pulse is adjustable by digital delay generators. The camera system is arranged in a shadowgraphy configuration, ${ }^{13}$ similar to Ref. 6 , but without the pinhole. High-speed photographic film (Polaroid Type 47) is used. The dye laser diagnostic utilized neutral density attenuators in the path after the beam passed through the plume; to attenuate the bright flash from the laser ablationinduced plasma at high fluences. The ablation target vessel is evacuated by a turbomolecular pump. After exposure to air the target was treated by about 100 excimer laser pulses in vacuum to remove any aluminum-oxide coating. In these experiments the dye laser wavelength was chosen to excite the ground-state neutral aluminum $3^{2} P_{1 / 2}-4^{2} S_{1 / 2}$ transition at $394.4 \mathrm{~nm}$. (This line was utilized in previous

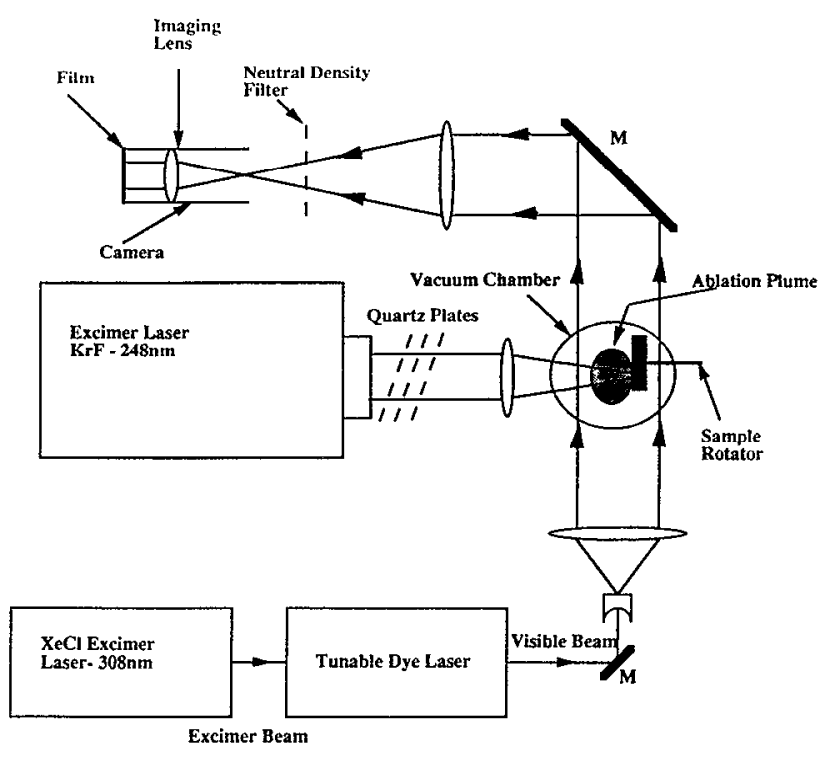

FIG. 1. Experimental configuration. 


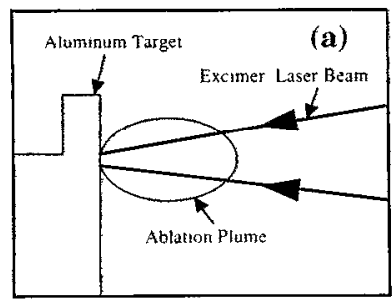

(b):
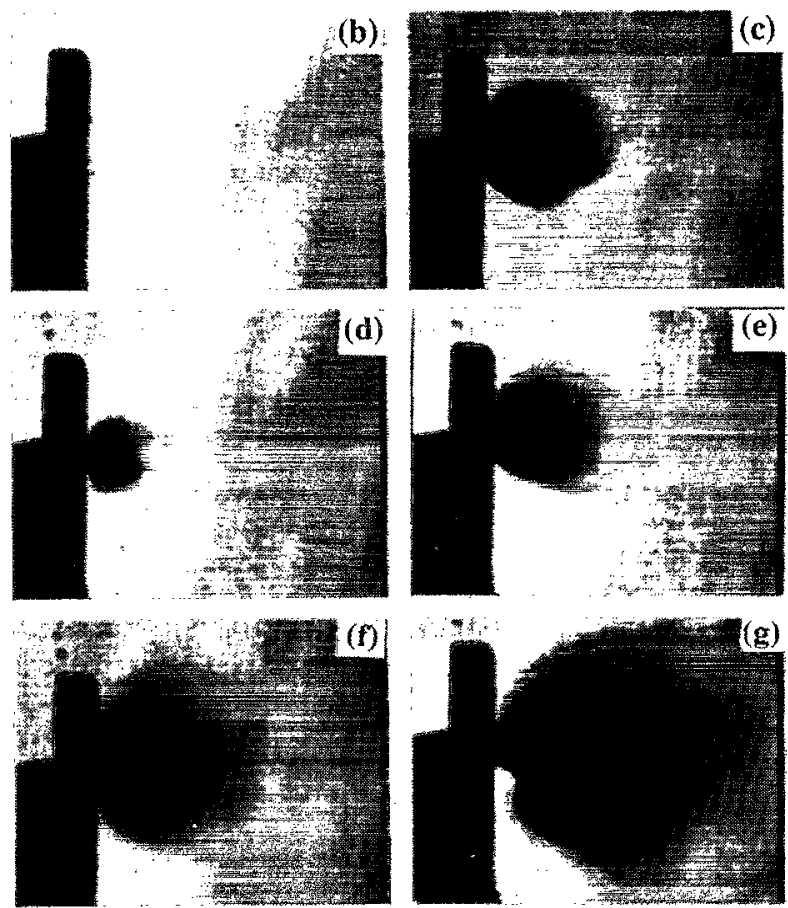

FIG. 2, (a) Geometry of laser ablation depicted in photographs, (b) and (c) Dye laser absorption photographs taken at 300 ns with an excimer laser fluence of $7 \mathrm{~J} / \mathrm{cm}^{2}$ : (b) photograph with dye laser detuned from resonance ( $\Delta \lambda=0.1 \mathrm{~nm}$ off resonance) wavelength; (c) Dye laser resonance absorption photograph with dye laser tuned to resonance condition; (d) - (g) Dye laser resonance absorption pholographs with dye laser tuned to resonance condition; excimer laser fuence of $3.7 \mathrm{~J} / \mathrm{cm}^{2}$; delay times between excimer laser pulse and dye laser pulse: (d) $340 \mathrm{~ns}$, (e) 460 $\mathrm{ns}$, (f) $540 \mathrm{~ns}$, and (g) $1.04 \mu \mathrm{s}$.

laser-induced fluorescence experiments of laser-ablated $\mathrm{Al}_{2} \mathrm{O}_{3}$ in vacuum. ${ }^{4}$ )

Figure 2 presents data for excimer laser ablation of aluminum in vacuum, where each photograph represents a single pulse from the excimer and dye lasers with a preset delay. Figure 2 (b) shows a dye laser absorption photograph for nonresonant ( $\Delta \lambda=0.1 \mathrm{~nm}$ off resonance) dye laser wavelength, taken $300 \mathrm{~ns}$ after the excimer laser pulse of fluence $7 \mathrm{~J} / \mathrm{cm}^{2}$; the film is completely exposed except for the target, with no visible plume. Figure $2(\mathrm{c})$ is a plume photograph taken with the dye laser tuned on resonance at the same delay time and fiuence as Fig. 2(b). Figures $2(\mathrm{~d})-2(\mathrm{~g})$ show a sequence of pulses in which the dye laser was tuned to the resonance absorption line at different delay times for an excimer laser fluence of 3.7 $\mathrm{J} / \mathrm{cm}^{2}$. The aluminum neutral plume can be seen to expand away from the excimer laser focal spot in both parallel and perpendicular directions from the surface. Dye laser absorption photographs near the ablated surface (at KrF fluence of $6-7 \mathrm{~J} / \mathrm{cm}^{2}$ ) could be obtained for wavelength shifts of $\pm 0.1 \mathrm{~nm}$ at a delay time of $200 \mathrm{~ns}$ after the excimer laser pulse, but wavelength shifts of only $\pm 0.01 \mathrm{~nm}$ at $1 \mu \mathrm{s}$ delay caused the pratterns to disappear from photos. This diagnostic linewidth may be related to peak density and possibly to plume temperature. Up to $90 \%$ maximum dye laser absorption by the plume was measured using a $p-i=n$ diode with an excimer laser fluence of $4.5 \mathrm{~J} / \mathrm{cm}^{2}$.

For gas phase expansion in vacuum the maximum particle velocity, $v_{m}$ is expected to scale as ${ }^{14}$

$$
v_{m}=2 c_{\alpha} /(\gamma-1) \text {, }
$$

and

$$
c_{0}=(\gamma k T / M)^{1 / 2},
$$

where $c_{0}$ is the initial sound speed, $\gamma(1.7)$ is the ratio of specific heats, $T$ is the temperature before expansion, and $M$ is the atomic rnass of aluminum. Using the measured velocity of the fastest particles at $2.6 \mathrm{~J} / \mathrm{cm}^{2}$ and solving for $c_{0}$, one can estimate an initial neutral aluminum temperature of about $1-2 \mathrm{eV}$. These temperatures suggest that a laser-heated plasma exists near the surface ${ }^{3,4}$ at early times. The ionization fraction of the ablation plume at early times

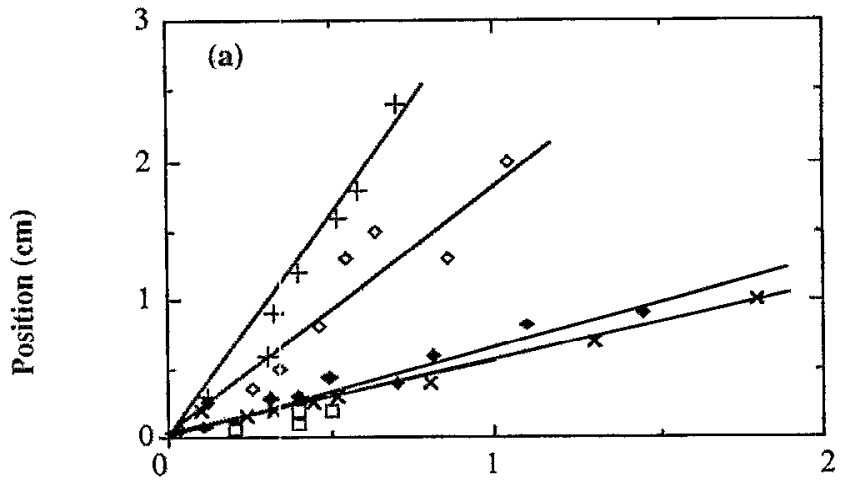

Time ( $\mu s)$

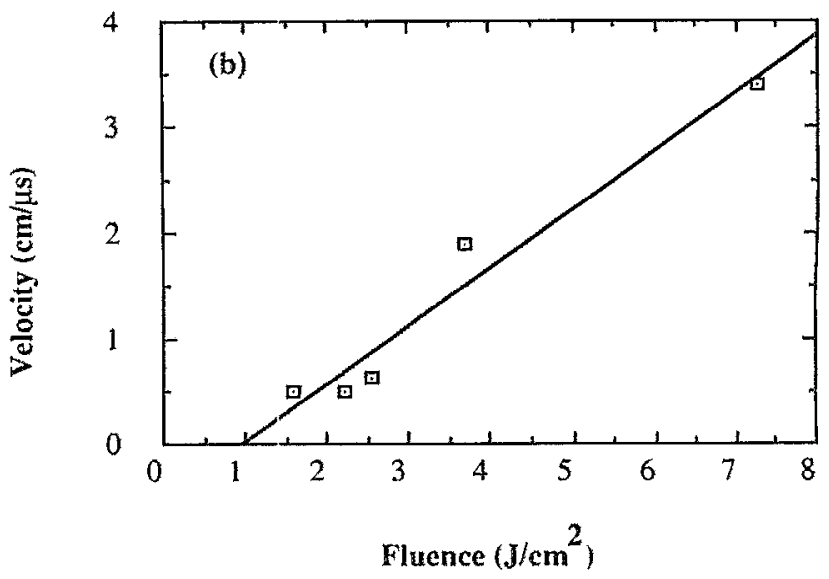

FIG. 3. (a) Position of plume edge measured by dye laser resonance absorption photography as a function of delay time between the excimer laser pulse and the dye laser pulse for different excimer laser fluences: (+) $7.2 \mathrm{~J} / \mathrm{cm}^{2} ;(\diamond) 3.7 \mathrm{~J} / \mathrm{cm}^{2} ;(\diamond) 2.6 \mathrm{~J} / \mathrm{cm}^{2} ;(\times) 2.2 \mathrm{~J} / \mathrm{cm}^{2} ;$ (口) 1.6 $\mathrm{J} / \mathrm{cm}^{2}$. (b) Expansion velocities of aluminum neutral atom plumes perpendicular to the surface, as a function of excimer laser fluence. Velocities obtained by taking the slope of the data in (a). 

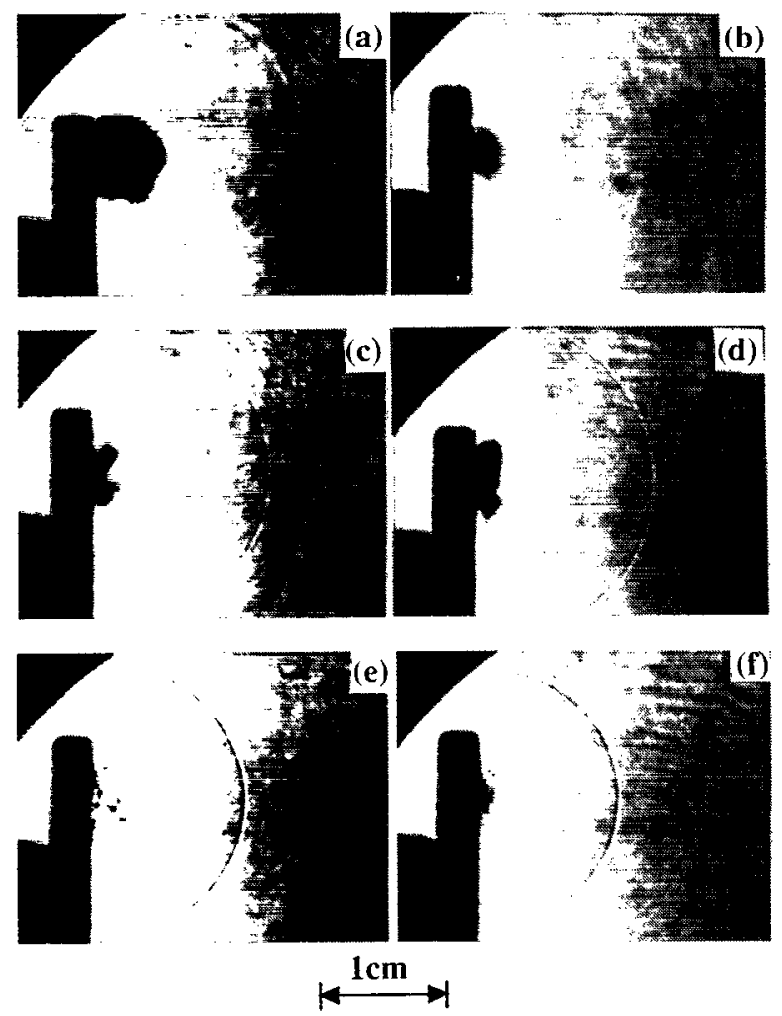

FIG. 4. Dye laser resonance absorption photographs of laser-ablated aluminum neutral atom plumes in gas backgrounds: at excimer laser fluence of $4 \mathrm{~J} / \mathrm{cm}^{2}$. Air data in left-hand column $(a, c, e)$; argon data in right-hand column (b,d.f). Data taken at pressures of 50 Torr (a and b), 200 Torr (c and $d$ ), and 760 Torr (e and $f$ ). Delay of $23 \mu$ s between excimer laser pulse and dye laser pulse.

exceeds that of the late time plume, since recombination occurs very rapidly (on ns time scales) after the laser pulse.

A series of dye laser resonance absorption photographs were taken over a range of delay times after the $\mathrm{KrF}$ excimer laser pulse to measure the expansion of the neutralaluminum plume in vacuum, as shown in Fig. 3(a). The edge of the plume was obtained at the midpoint in the black-to-white transition. From our measured film response the black-to-white transition corresponded to attenuation by a factor of about 30 .

Figure $3(\mathrm{~b})$ gives a plot of expansion velocities measured as a function of excimer laser fluence, in the direction perpendicular to the sample surface. The plume velocity scales linearly with laser fluence. Plume velocities $\left(0.5 \times 10^{6} \mathrm{~cm} / \mathrm{s}\right)$ at low fluence $\left(1-2 \mathrm{~J} / \mathrm{cm}^{2}\right)$ are in reasonable agreement with the previous LIF measurements of neutral aluminum made for low fluence excimer laser ablation of alumina in vacuum. ${ }^{4}$ The neutral aluminum streaming velocities at higher fluence $\left(7.2 \mathrm{~J} / \mathrm{cm}^{2}\right)$ reach about $3.4 \times 10^{6} \mathrm{~cm} / \mathrm{s}$.

A second experiment was performed to utilize dye la- ser resonance absorption photography to diagnose excimer laser ablation of aluminum in both argon and air at pressures ranging from 50 to 760 Torr. Typical DLRA photographs are shown in Fig. 4 for the dye laser pulsed $23 \mu \mathrm{s}$ after the excimer laser. At pressures of 50 Torr, the plume expansion can be seen to be much slower than that in vacuum for both gases. Data taken at 200 Torr shows that a neutral aluminum plume persists at the surface, but expands very little in the background gas. Spherical shock waves with $0.3 \mathrm{~mm}$ thickness propagate ahead of the plume. At 760 Torr, turbulent mixing of aluminum with background gas occurs near the surface for argon and air; this might be expected from the Rayleigh-Taylor instability because the atomic mass of aluminum is lower than Ar, $\mathrm{N}_{2}$, or $\mathrm{O}_{2}$. Differences between DLRA photographs of laser-ablated aluminum in argon versus air could be due to oxidation. ${ }^{15}$

In summary, dye laser resonance absorption photography has been shown to be a valuable species resolved diagnostic for laser ablated aluminum neutral plumes in both vacuum and gaseous environments. We have also found that this technique is applicable with other absorption lines for ablated aluminum, other elements (iron), and molecules ( $\mathrm{CN}$ from ablation of polyimide).

We thank R. A. Lindley for experimental assistance. This research was supported by National Science Foundation grant No. ECS-8351837 and by Los Alamos National Laboratorics.

' J. A. Sell, D. M. Heffelfinger, P. Ventzek, and R. M. Gilgenbach, Appl. Phys. Lett. 55, 2435 (1989)

${ }^{2}$ C. L. Enloe, R. M. Gilgenbach, and J. Meachum, Rev. Sci. Instrum. 58, 1597 (1987).

${ }^{3}$ R. E. Walkup, J. M. Jasinski, and R. W. Dreyfus, Appl. Phys. Lett. 48, 1960 (1986).

${ }^{4}$ R. W. Dreyfus, R. Kelly, and R. E. Walkup, Appl. Phys. Lett. 49, 1478 (1986).

${ }^{5}$ D. B. Geohagen and D. N. Mashburn, Appl. Phys. Lett. 55, 2345 (1989).

${ }^{6}$ P. L. G. Ventzek, R. M. Gilgenbach, J. A. Sell, and D. M. Heffelfinger, J. Appl. Phys. 68, 965 (1990).

${ }^{7}$ L. D. Horton and R. M. Gilgenbach, Appl. Phys. Lett. 43, 1010 (1983).

${ }^{8}$ R. Srinivasan, K. G. Casey, B. Braren, and M. Yeh, J. Appl. Phys. 67, 1604 (1990); R. Srinivasan, B. Braren, K. Casey, and M. Yeh, Appl. Phys. Lett. 55, 2790 (1989).

${ }^{9}$ P. E. Dyer, A. Issa, and P. H. Key, Appl. Phys. Lett. 57, 186 (1990); P. E. Dyer and J. Sidhu, J. Appl. Phys. 64, 4657 (1988).

${ }^{10} \mathrm{C}$. Girault, D. Damiani, J. Aubreton, and A. Catherinot, Appl. Phys. Lett. 55, 182 (1989).

${ }^{11}$ Q. Y. Ying, D. T. Shaw, and H. S. Kwok, Appl. Phys. Lett. 53, 1762 (1988).

${ }^{12}$ O. Auciello, S. Athavale, O. E. Hawkins, M. Sito, A. F. Schreiner, and N. Biunno, Appl. Phys. Lett. 53, 72 (1988).

${ }^{13}$ K. Horioka, N. Tazima, and K. Kasuya, Rev. Sci. Instrum. 61, 610 (1990).

${ }^{14}$ Y. B. Zel'dovich and Yu. P. Raizer, Physics of Shock Waves and High Temperature Hydrodynamic Phenomena (Academic, New York, 1966), Vol. 1.

${ }^{15}$ A. Fontijn, W. Felder, and J. J. Houghton, Chem. Phys. Lett. 27, 365 (1974). 